\title{
Design of a dual-band indoor antenna with orthogonal bi-directional radiation pattern
}

\author{
Ho-Yu Lin ${ }^{\text {a) }}$ and Masayuki Nakano \\ KDDI R\&D Laboratories Inc., \\ 2-1-15 Ohara, Fujimino-shi, Saitama 356-8502, Japan \\ a)ho-lin@kddilabs.jp
}

Abstract: In recent years, MIMO (Multi-Input Multi-Output) transmission technique has attracted increasing attention, as it can satisfy the need for exponential increase in data traffic for mobile communications, especially when used for indoor areas. In this paper, an antenna design is proposed that can operate in the $2.6 \mathrm{GHz}$ and $3.5 \mathrm{GHz}$ bands and produce an orthogonal bidirectional radiation pattern. Some characteristics of the proposed antenna, such as reflection coefficient $\left(S_{11}\right)$, transmission coefficient $\left(S_{21}\right)$ and far-field radiation pattern are simulated and evaluated by means of FDTD (finitedifference time-domain) methods. The results show that $S_{11}$ and $S_{21}$ are below $-10 \mathrm{~dB}$ and $-15 \mathrm{~dB}$, and moreover, the MIMO performance can probably be improved because the difference between the two directional gain levels is achieved by approximately $15 \mathrm{~dB}$ when four slits are etched into the ground plane.

Keywords: indoor antenna, base station antenna, MIMO, dual band Classification: Antennas and Propagation

\section{References}

[1] M. Nakano, et al., "Small-sized polarization diversity antenna for cellular base stations," Proc. of the 1997 IEICE Society Conference, B-1-42, Aug. 17, 2014.

[2] M. Nakano and H. Arai, "Orthogonal polarization base station antenna technology at cellular systems and system evaluation," IEICE Trans. Commun., vol. E96-B, no. 1, pp. 1-15, Jan. 2013.

[3] K. Cho, et al., "Influence of terminal MIMO antenna polarization on MIMO transmission performance using dual polarized antenna," Technical Report of IEICE, AP2011-5, pp. 81-84, May 2011.

[4] C.-L. Yang, J. F. Mastarone, and W. J. Chappell, "Directional antennas for angular diversity in wireless sensor networks," IEEE Antennas and Propagation Society International Symposium. vol. 4A, pp. 263-266, 2005. DOI:10.1109/ APS.2005.1552638

[5] D. Kitchener, M. S. Smith, J. E. J. Dalley, and R. R. Thomas, "Low cost diversity antennas for low power wireless base stations," 10th International Conference on Antennas and Propagation, vol. 1, pp. 445-447, Apr. 1997. DOI:10.1049/cp:19970291

[6] T. Ihara and K. Tsunekawa, "Indoor base station antennas for mobile 
communications with rounded semi-circular element," Technical Report of IEICE, AP97-70, pp. 1-6, July 1997.

[7] K. L. Lau and K. M. Luk, "A wide-band monopolar wire-patch antenna for indoor base station applications," IEEE Antennas Wireless Propag. Lett., vol. 4, pp. 155-157, 2005. DOI:10.1109/LAWP.2005.847432

[8] Z.-Y. Zhang, G. Fu, W.-J. Wu, J. Lei, and S.-X. Gong, “A wideband dual-sleeve monopole antenna for indoor base station application," IEEE Antennas Wireless Propag. Lett., vol. 10, pp. 45-48, 2011. DOI:10.1109/LAWP.2011. 2108255

[9] S. Sun, M. Cheng, S. Lu, and J. Lin, "Compact MIMO PIFA for LTE/WWAN operation in the mobile application," 2014 3rd Asia-Pacific Conference on Antennas and Propagation, pp. 26-28, 2014. DOI:10.1109/APCAP.2014. 6992401

[10] S. Wang and Z. Du, "Decoupled dual-antenna system using crossed neutralization lines for LTE/WWAN smartphone applications," IEEE Antennas Wireless Propag. Lett., vol. 14, pp. 523-526, 2014. DOI:10.1109/LAWP.2014. 2371020

[11] CST Microwave STUDIO, [Online] http://www.cst.com.

[12] J. D. Kraus, et al., Antennas, Third Edition, McGRAW-HILL, Singapore, 2003.

\section{Introduction}

In recent years, the MIMO (Multi-Input Multi-Output) transmission technique has attracted increasing attention as it can satisfy the need for the exponential increase in data traffic required by mobile communications, especially for indoor and densely populated areas. In general, base stations use diversity reception to minimize the effect of Rayleigh fading on the received signal. Polarization spatial diversity techniques are the most common forms of diversity reception $[1,2,3]$. Another form of diversity, angular diversity techniques are also effective at mitigating multipath situations [4], because multiple antennas are oriented in different directions, but multiple antennas are required to form such systems. However, for indoor base stations, it is considered that a device must be compact, and its location should be optimized for maximum antenna coverage.

A number of studies on indoor base station antennas have been published in journals and conference papers. According to [5], in 1997 the main design target for an indoor base station antenna and the effect of the ground plane were introduced. A design for a cylindrical monopole antenna was proposed to reduce the physical antenna size [6]. In order to enhance the bandwidth, a dual-sleeve antenna structure and the use of a shorting wire were proposed [7] and [8].

In this paper, we propose a novel compact indoor base station antenna that is designed to operate in the $2.6 \mathrm{GHz}$ and $3.5 \mathrm{GHz}$ bands. The $S_{11}$ and $S_{21}$ are satisfying the requirement of $S_{11}<-10 \mathrm{~dB}$ and $S_{21}<-15 \mathrm{~dB}$ in MIMO system [9] and [10], and moreover, the proposed antenna has an orthogonal bi-directional radiation pattern for each frequency band, which means that angular diversity gains could be improved to a practically useful extent in a multipath environment. Moreover, the MIMO performance can probably be improved because the difference between the two directional gain level can be achieved by approximately 
$15 \mathrm{~dB}$ when we etched four slits into ground plane. The simulation was performed with CST Microwave STUDIO [11].

\section{Antenna structure}

\subsection{Basic principles of radiation patterns}

Fig. 1 illustrates the radiation pattern of two isotropic sources in the same phase and opposite phase, and the difference between the two directional gain levels on an orthogonal bi-directional radiation pattern. Fig. 1(a) shows the two isotropic sources separated by a distance $d$ and located along the $y$-axis. As we know, beam-width and main-beam direction can be controlled by changing the signal amplitude and phase of each antenna [12]. For instance, when two isotropic sources have the same amplitude and phase (Phase difference, $\delta=0$-degree), the mainbeam of the vector sum of the fields is orthogonal to the $x$-axis direction. Similarly, when they have the same amplitude but opposite phase (Phase difference, $\delta=$ 180-degree), the main-beam is in the $y$-axis direction. The vector addition of the fields as mentioned above can be expressed as

$$
\begin{aligned}
& E=2 E_{0} \cos \left(\frac{d}{2} \cos \theta\right) \\
& E=2 j E_{0} \sin \left(\frac{d}{2} \cos \theta\right)
\end{aligned}
$$

where $d=\lambda / 2$ and the amplitude of $2 E_{0}$ and $2 j E_{0}=1$ are considered as a special case. We should note that the field pattern in the opposite phase given by (2) is a relatively broad figure-eight with the maximum field.

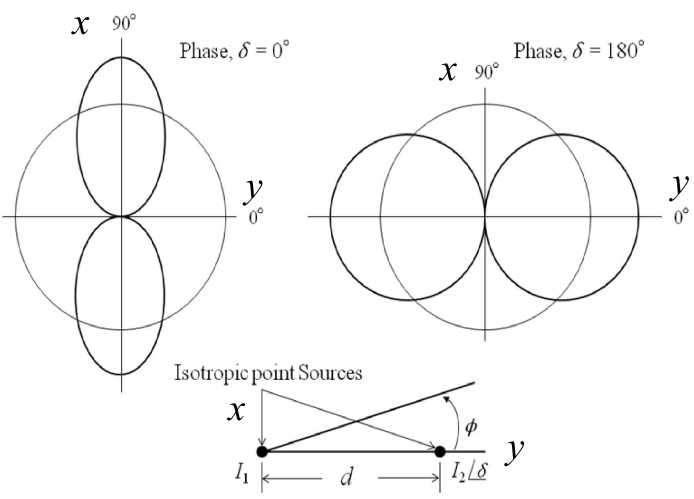

(a) Field pattern of two isotropic sources

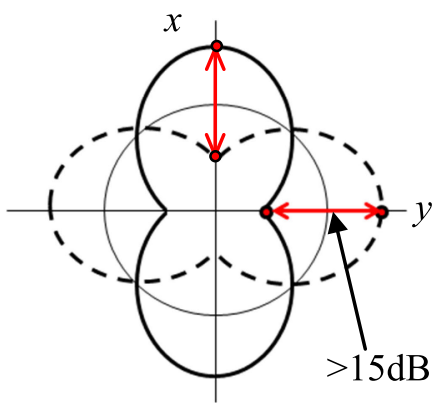

(b) Orthogonal bi-directional radiation pattern

Fig. 1. Schematic of the radiation pattern and image of an orthogonal bi-directional radiation pattern.

According to this concept, when we set the phases of two isotropic sources to be the same or opposite by use of 180-degree hybrid couplers, an orthogonal bidirectional radiation pattern can be formed to extend simultaneously in the $x$-axis and $y$-axis directions. However, it should be noted that the overlapping patterns also cause the undesirable high correlated or correlated MIMO signals to affect MIMO performance. Fig. 1(b) shows an image of the desirable radiation pattern. From the image, the difference between the two directional gain level should be large in order 
to reduce the overlapping on the orthogonal bi-directional radiation pattern, that meaning the MIMO performance can probably be improved. Therefore, we considered that the difference should be at least $15 \mathrm{~dB}$ in order to improve MIMO performance. In next section, the structure of the proposed antenna and the detailed simulation results are shown and discussed.

\subsection{Antenna structure and simulation result}

Fig. 2(a) shows a prototype model of the vertical polarized antenna. Two pairs of monopole antennas, both with a diameter of $2 \mathrm{~mm}$, are mounted on a ground plane and located along the $y$-axis. $2.6 \mathrm{GHz}$ and $3.5 \mathrm{GHz}$ antenna elements are separated by a distance $d_{1}$ of $0.5 \lambda$ and $d_{2}$ of $0.59 \lambda$, respectively. The circular ground plane is formed from an indoor base station for practical use, and its diameter and thickness are set to $100 \mathrm{~mm}$ and $0.5 \mathrm{~mm}$. In order to improve the input impedance matching, we introduced an L-type matching-pin that is designed to connect the monopole antenna for $3.5 \mathrm{GHz}$ with the ground plane as shown in Fig. 2(b). The height $(h)$ and distance $(w)$ between matching-pin and monopole antenna are $5.5 \mathrm{~mm}$ and $1 \mathrm{~mm}$. Four slits are etched into the ground plane and symmetrically arranged to improve the diversity performance of the proposed antenna and the width and length $(l)$ of each slit are $1 \mathrm{~mm}$ and $35 \mathrm{~mm}$. Gap feeding is set at the bottom of each antenna. The parameters of $w$ and $h$ can be changed to obtain the optimized result for impedance matching. For the radiation pattern, the optimized result can be obtained by tunning the width and length of slit and $d_{2}$.

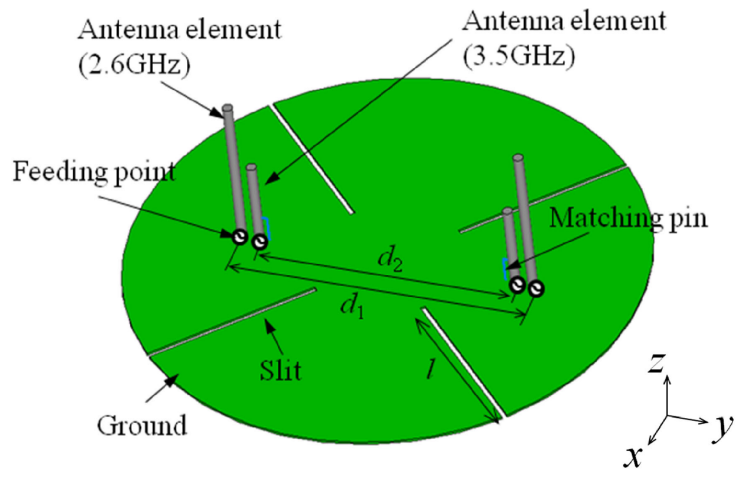

(a) Overview

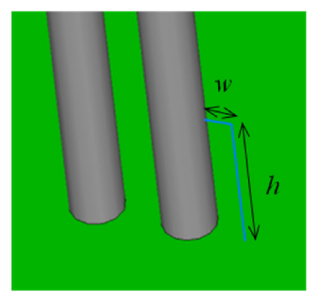

(b) Matching-pin

Fig. 2. Prototype of the proposed antenna structure.

Fig. 3 shows the simulation results for the $S$-parameters and far-field radiation pattern. From the results show in Fig. 3(a), when the matching-pin is not used, it can be seen that $S_{11}$ in the $3.5 \mathrm{GHz}$ band does not satisfy the required value of $-10 \mathrm{~dB}$, possibly because the antennas are close to each other and the coupling between them becomes strong. $S_{21}$ at $2.6 \mathrm{GHz}$ is $-17 \mathrm{~dB}$, which satisfies the $-15 \mathrm{~dB}$ requirement, but at $3.5 \mathrm{GHz}$ it is $-13 \mathrm{~dB}$ and needs to be improved. Fig. 3(b) shows the results with the matching-pin, the input impedance in the $3.5 \mathrm{GHz}$ band can be improved $\left(S_{11}<-10 \mathrm{~dB}\right)$ and $S_{21}$ at the $2.6 \mathrm{GHz}$ and $3.5 \mathrm{GHz}$ is $-15 \mathrm{~dB}$ and $-24 \mathrm{~dB}$, which satisfies the required $-15 \mathrm{~dB}$. The bandwidth of the proposed antenna at $2.6 \mathrm{GHz}$ and $3.5 \mathrm{GHz}$ are $50 \%$ and $17 \%$, respectively. 
Fig. 3(c) and (d) plot the radiation pattern without four slits. From the result, although the proposed antenna seems to have an orthogonal bi-directional radiation pattern, the difference between the two directional gain levels is $10.8 \mathrm{~dB}$ (in the $x$ direction) and $20.6 \mathrm{~dB}$ (in the $y$ direction) at the $2.6 \mathrm{GHz}, 22.5 \mathrm{~dB}$ (in the $x$ direction) and $14.2 \mathrm{~dB}$ (in the $y$ direction) at the $3.5 \mathrm{GHz}$ band, so some results need to be improved to satisfy the $15 \mathrm{~dB}$ requirement. Fig. 3(e) and (f) show the radiation pattern when the four slits are etched into ground plane. From the results, the difference between the two directional gain levels is $26.1 \mathrm{~dB}$ (in the $x$ direction) and $25.6 \mathrm{~dB}$ (in the $y$ direction) at the $2.6 \mathrm{GHz}$ band, and $22.8 \mathrm{~dB}$ (in the $x$ direction) and $16.9 \mathrm{~dB}$ (in the $y$ direction) at the $3.5 \mathrm{GHz}$ band, all results fulfill the $15 \mathrm{~dB}$ requirement by the addition of the four slits. For the opposite phase case at $2.6 \mathrm{GHz}$ the difference between two directional gain levels has been improved from $10.8 \mathrm{~dB}$ to $26.5 \mathrm{~dB}$ (in the $y$ direction), the amount of improvement is approximately $15 \mathrm{~dB}$.

Fig. 3(g) and (h) present the excited surface current distribution, obtained from the simulation of the ground plane of the proposed antenna, for the opposite phase case at $2.6 \mathrm{GHz}$. Comparison of $(\mathrm{g})$ and $(\mathrm{h})$ shows that when four slits are etched into the ground plane, the current in the $x$ direction is lower than it is without the slits. This is thought to result from currents flowing $\left(\mathrm{I}_{1}\right.$ and $\left.\mathrm{I}_{2}\right)$ along the slits that mutually cancel, making that the main reason for the improvement in the difference between the two directional gain levels in orthogonal bi-directional radiation patterns.

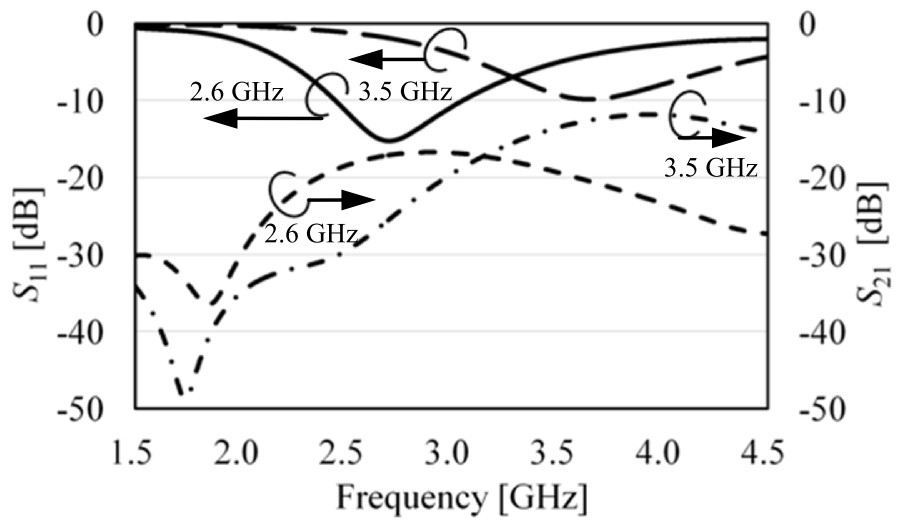

(a) $S$-parameter (w/o matching-pin)

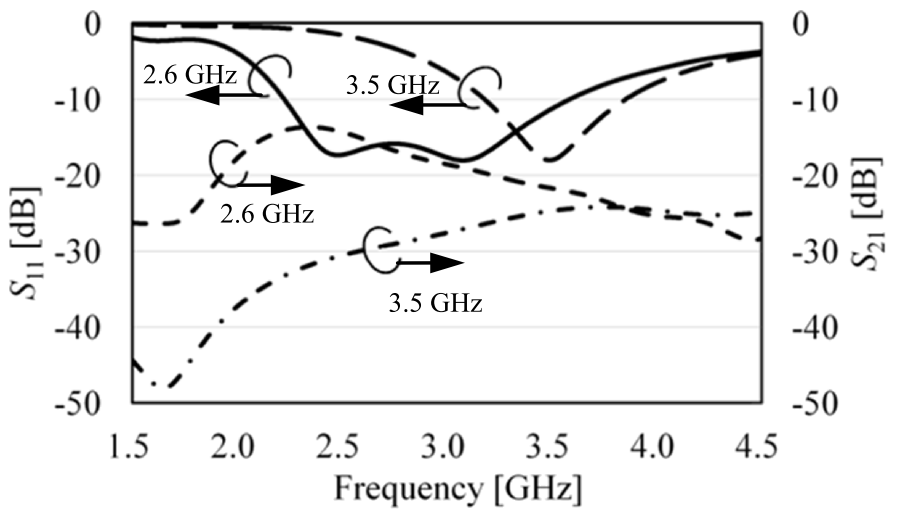




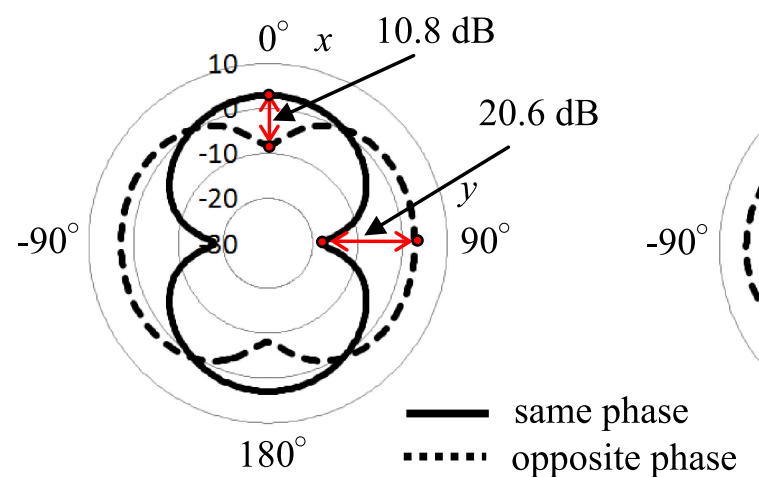

(c) Radiation pattern at $2.6 \mathrm{GHz}$ (w/o slit)

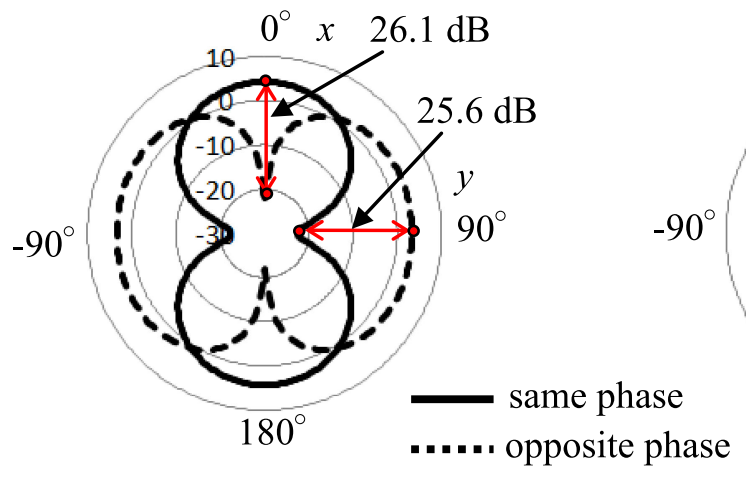

(e) Radiation pattern at $2.6 \mathrm{GHz}$ (with slit)

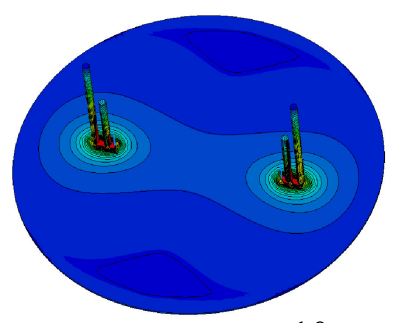

10

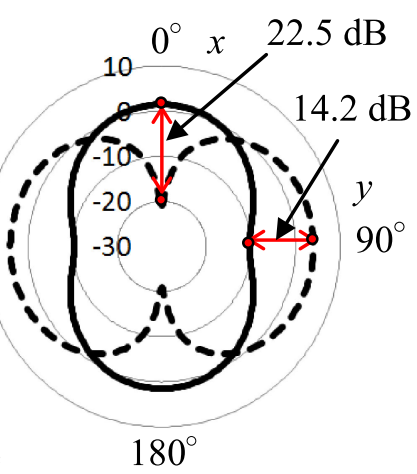

(d) Radiation pattern at $3.5 \mathrm{GHz}$ (w/o slit)

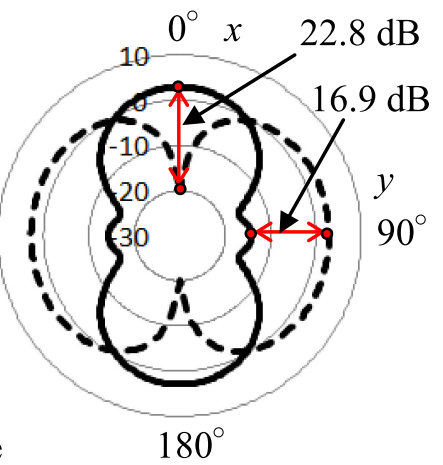

(f) Radiation pattern at $3.5 \mathrm{GHz}$ (with slit)

(g) Current distribution (w/o slit)

5

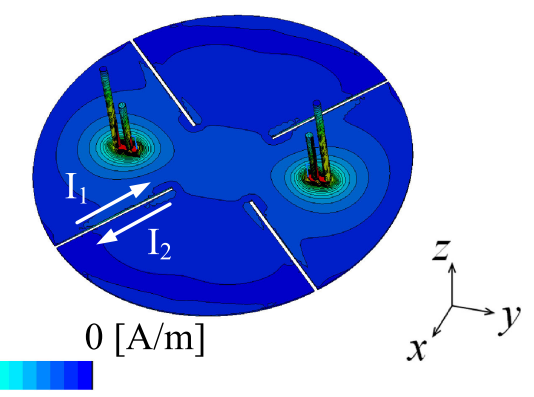

(h) Current distribution (with slit)

Fig. 3. Simulation results of the proposed antenna

\section{Conclusions}

In this paper, we propose a novel compact indoor base station antenna with an orthogonal bi-directional radiation pattern for dual-band operation at $2.6 \mathrm{GHz}$ and 3.5 GHz. The simulated results indicate that input impedance in the $3.5 \mathrm{GHz}$ band can be improved by adding a matching-pin, bringing the $S_{11}$ below the required value of $-10 \mathrm{~dB}$. Moreover, the MIMO performance can probably be improved because the difference between the two directional gain levels is achieved by approximately $15 \mathrm{~dB}$ when four slits are etched into the ground plane. The proposed antenna's structure is simple and compact, which will be useful when designing high-performance base station antennas. In future work, the estimation of MIMO capacity will be implemented and an experiment will be carried out to test the simulated results presented here for the proposed antenna. 